\title{
A Gaussian Model for the Membrane of Red Blood Cells with Cytoskeletal Defects
}

\author{
Cyril Dubus $^{1,2}$ and Jean-Baptiste Fournier ${ }^{1,2, \text {, }}$ \\ ${ }^{1}$ Laboratoire Matière et Systèmes Complexes (MSC), \\ UMR 7057 CNRS \&S Université Paris 7, 2 place Jussieu, F-75251 Paris Cedex 05, France \\ ${ }^{2}$ Laboratoire de Physico-Chimie Théorique, UMR CNRS 7083 \& ESPCI, \\ 10 rue Vauquelin, F-75231 Paris Cedex 05, France
}

(Dated: October 16, 2018)

\begin{abstract}
We study a Gaussian model of the membrane of red blood cells: a "phantom" triangular network of springs attached at its vertices to a fluid bilayer with curvature elasticity and tension. We calculate its fluctuation spectrum and we discuss the different regimes and non-monotonic features, including the precise crossover at the mesh size between the already known limits with two different tensions and the renormalisation of the bending rigidity at low wavevectors. We also show that the non-diagonal correlations reveal, in "dark field", the cytoskeletal defects. As a first step toward a non-invasive defect spectroscopy, the specific case of lacking bonds is studied numerically and analytically.
\end{abstract}

PACS numbers: 87.16.Gj, 87.16.Dg, 61.72.Hh

The outer walls of biological cells are fluid bilayer membranes made by the self-assembly of lipid molecules in water [1]. For about three decades, the physical properties of these two-dimensional fluid surfaces, including elasticity, thermal fluctuations, phase behavior, topology, shape polymorphism, etc., have been thoroughly investigated in model systems [2, 3, 4]. In real cells, membranes host a large number of proteins and other inclusions, and they are attached to a network of filaments called the cytoskeleton [1]. In most cases this network is three-dimensional. In the case of red blood cells (RBC's), however, the cytoskeleton is a triangular two-dimensional network of flexible spectrin filaments attached to the membrane by proteins located at its vertices [5, 6, 7]. Recent experimental and theoretical studies have focused on the shape elasticity [8, 9, 10, 11, 12] and fluctuation properties 13, 14, 15, 16, 17] of these composite membranes. Up to now, the available theoretical models describing the RBC membrane fluctuations either are meanfield [15] or describe the cytoskeleton as a fixed plane producing a checkerboard (square) modulated potential [16]. In order to obtain a better description, it is essential to release the assumption of a fixed cytoskeletal plane and to allow for coupled fluctuations of the membrane and the cytoskeleton. It is also desirable to take into account the triangular nature of the cytoskeleton and to determine the effects of the cytoskeletal defects [17, 18].

In this paper, we propose a simple gaussian model describing the composite membrane of RBC's. It allows to calculate explicitely the whole fluctuation spectrum, even in the presence of cytoskeletal defects, such as lacking bonds and seven-fold or five-fold defects. We model the composite membrane (Fig. 1) as a triangular network of harmonic springs attached at its vertices to a fluid membrane described by a curvature Hamiltonian plus surface tension [19]. The only coupling between the two subsystems is the constraint that the cytoskeleton vertices lie within the membrane surface. As in model polymers [20], the gaussian character arises from neglecting the excluded volume between the

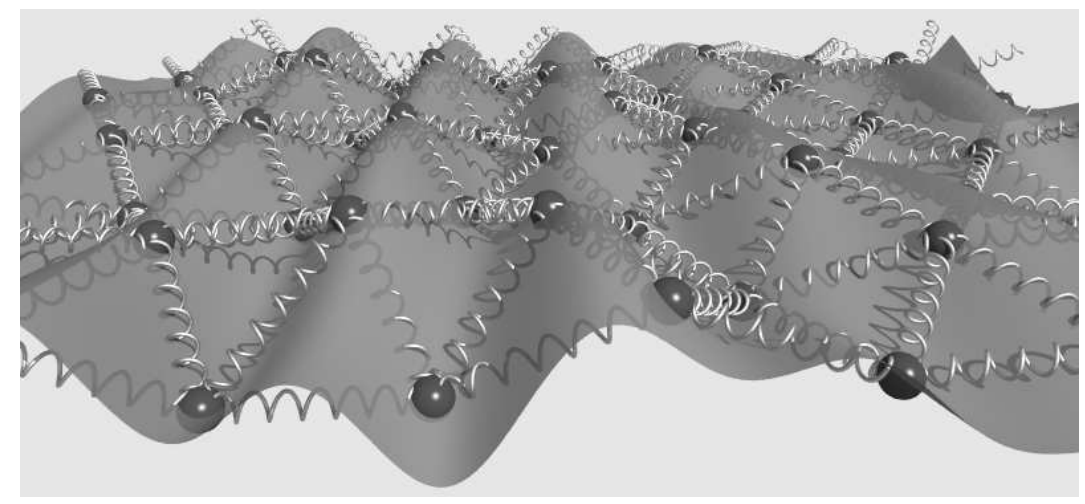

FIG. 1: Sketch of the model red blood cell (RBC) membrane.

*author for correspondance 
by $\sqrt{\kappa / \beta} \sigma^{-1}$ and $\mu_{n}$ by $\sigma$. Unless otherwise specified, all quantities are now dimensionless. We thus have $\mathcal{H}_{0}=$ $\sum_{\mathbf{q}, \mathbf{k}} \frac{1}{2} h_{\mathbf{q}} G_{\mathbf{q}, \mathbf{k}}^{-1} h_{\mathbf{k}}$ where $G_{\mathbf{q}, \mathbf{k}}=\delta_{\mathbf{q}+\mathbf{k}} /\left(\mathbf{q}^{2}+\mathbf{q}^{4}\right)$ and $\mathbf{q}^{2}=q^{\alpha} q_{\alpha}$, yielding the correlation function of the bare membrane:

$$
G(\mathbf{x})=\langle h(\mathbf{0}) h(\mathbf{x})\rangle=\frac{1}{\sqrt{g} L_{1} L_{2}} \sum_{\mathbf{q}} \frac{e^{i q_{\alpha} x^{\alpha}}}{\mathbf{q}^{2}+\mathbf{q}^{4}} .
$$

For an infinite membrane, this gives a Bessel function of the second kind: $G(\mathbf{x})=-\frac{1}{4} Y_{0}(|\mathbf{x}|)$. Because $\mathcal{H}$ is not diagonal in q-space due to the dependence in $\mathbf{R}_{n}$ and $\overline{\mathbf{R}}_{n}$, it cannot be directly inverted to yield the full correlation function $G(\mathbf{x})=\langle h(\mathbf{0}) h(\mathbf{x})\rangle$. To proceed, we add an external field, $\mathcal{H} \rightarrow \mathcal{H}+\sum_{\mathbf{q}} h_{\mathbf{q}} f_{-\mathbf{q}}$, and we calculate $\left\langle h_{\mathbf{q}} h_{\mathbf{k}}\right\rangle=$ $-\partial^{2} F /\left.\left(\partial f_{-\mathbf{q}} \partial f_{-\mathbf{k}}\right)\right|_{f=0}$, where $F=-\ln \left[\int \mathcal{D}[h] \exp (-\mathcal{H})\right]$ is the free-energy. In order to integrate the membrane degrees of freedom, we rewrite $\exp (-\mathcal{V})$ with the help of auxialiary fields (one for each spring) as

$$
\int \prod_{n} d \phi_{n} \exp \sum_{n}\left(-\frac{\phi_{n}^{2}}{2 \mu_{n}}+i \phi_{n}\left[h\left(R_{n}^{\alpha}\right)-h\left(\bar{R}_{n}^{\alpha}\right)\right]\right) .
$$

Sums and products over $n$ (and further on $m$ ) implicitely run from 1 to $N$. Performing the gaussian integral over $h$ yields $\exp (-F)=\int \prod_{n} d \phi_{n} \exp (-\tilde{\mathcal{H}})$, where

$$
\tilde{\mathcal{H}}=\sum_{n} \frac{\phi_{n}^{2}}{2 \mu_{n}}-\frac{1}{2} \sum_{\mathbf{q}, \mathbf{k}}\left(S_{-\mathbf{q}}-f_{-\mathbf{q}}\right) G_{\mathbf{q}, \mathbf{k}}\left(S_{-\mathbf{k}}-f_{-\mathbf{k}}\right),
$$

in which $S_{\mathbf{q}}=\left(\sqrt{g} L_{1} L_{2}\right)^{-\frac{1}{2}} \sum_{n} i \phi_{n}\left(e^{-i q_{\alpha} R_{n}^{\alpha}}-e^{-i q_{\alpha} \bar{R}_{n}^{\alpha}}\right)$. The integral over the $\phi_{n}$ is again Gaussian, yielding $F[f]=$ $-\frac{1}{2} \sum_{\mathbf{q}, \mathbf{k}} f_{-\mathbf{q}} G_{\mathbf{q}, \mathbf{k}} f_{-\mathbf{k}}+\delta F[f]$, with

$$
\delta F[f]=\frac{1}{2} \sum_{n, m}\left(\sum_{\mathbf{q}} c_{\mathbf{q}}^{(n)} f_{-\mathbf{q}}\right) B_{n m}^{-1}\left(\sum_{\mathbf{k}} c_{\mathbf{k}}^{(n)} f_{-\mathbf{k}}\right),
$$

where

$$
\begin{aligned}
c_{\mathbf{q}}^{(n)} & =\frac{1}{\left(\sqrt{g} L_{1} L_{2}\right)^{\frac{1}{2}}} \frac{e^{-i q_{\alpha} R_{n}^{\alpha}}-e^{-i q_{\alpha} \bar{R}_{n}^{\alpha}}}{\mathbf{q}^{2}+\mathbf{q}^{4}}, \\
B_{n m} & =\frac{1}{\mu_{n}} \delta_{n m}+G\left(\mathbf{R}_{n}-\mathbf{R}_{m}\right)-G\left(\mathbf{R}_{n}-\overline{\mathbf{R}}_{m}\right)-G\left(\overline{\mathbf{R}}_{n}-\mathbf{R}_{m}\right)+G\left(\overline{\mathbf{R}}_{n}-\overline{\mathbf{R}}_{m}\right) .
\end{aligned}
$$

It follows that the full correlation function $\Gamma_{\mathbf{q}, \mathbf{k}}=\left\langle h_{\mathbf{q}} h_{\mathbf{k}}\right\rangle=G_{\mathbf{q}, \mathbf{k}}+\Delta \Gamma_{\mathbf{q}, \mathbf{k}}$ is given, with no approximation, by

$$
\Gamma_{\mathbf{q}, \mathbf{k}}=\frac{\delta_{\mathbf{q}+\mathbf{k}}}{\mathbf{q}^{2}+\mathbf{q}^{4}}-\sum_{n, m=1}^{N} c_{\mathbf{q}}^{(n)} B_{n m}^{-1} c_{\mathbf{k}}^{(m)} .
$$

For weak cytoskeletal strains, i.e., $\mu_{n}=\mathcal{O}(\mu) \ll 1$, we have $B_{n m}^{-1}=\mu_{n} \delta_{n m}+\mathcal{O}\left(\mu^{2}\right)$, and thus

$$
\Delta \Gamma_{\mathbf{q}, \mathbf{k}} \simeq-\frac{1}{\sqrt{g} L_{1} L_{2}} \sum_{n=1}^{N} \mu_{n} \frac{\psi_{n, \mathbf{q}} \psi_{n, \mathbf{k}}}{\left(\mathbf{q}^{2}+\mathbf{q}^{4}\right)\left(\mathbf{k}^{2}+\mathbf{k}^{4}\right)}
$$

where $\psi_{n, \mathbf{q}}=e^{-i \mathbf{q} \cdot \mathbf{R}_{n}}-e^{-i \mathbf{q} \cdot \overline{\mathbf{R}}_{n}}$. The fluctuation spectrum can thus be obtained analytically for weak strains even in presence of defects, or, for larger strains, numerically by simply inverting a $N \times N$ matrix.

Uniform cytoskeleton. - In the case of a uniform dilation or compression in the absence of defects, the points $\mathbf{R}_{n}$ and $\overline{\mathbf{R}}_{n}$ form a regular triangular lattice with $\mu_{n}=\mu, \forall n$, corresponding to a uniform meshsize $\xi$ such that $N=3 L_{1} L_{2} / \xi^{2}$. Thus, in dimensional units and for small values of $\mu / \sigma$, Eq. (10) gives for $\mathbf{k}=-\mathbf{q}$ the following fluctuation spectrum:

$$
\left\langle\left|h_{\mathbf{q}}\right|^{2}\right\rangle \simeq \frac{k_{\mathrm{B}} T}{\sigma \mathbf{q}^{2}+\kappa \mathbf{q}^{4}}-\frac{4 \mu g^{-\frac{1}{2}} \xi^{-2} k_{\mathrm{B}} T}{\left(\sigma \mathbf{q}^{2}+\kappa \mathbf{q}^{4}\right)^{2}} \sum_{j=0}^{2} \sin ^{2}\left[\frac{q \xi}{2} \cos \left(\theta-\frac{j \pi}{3}\right)\right],
$$




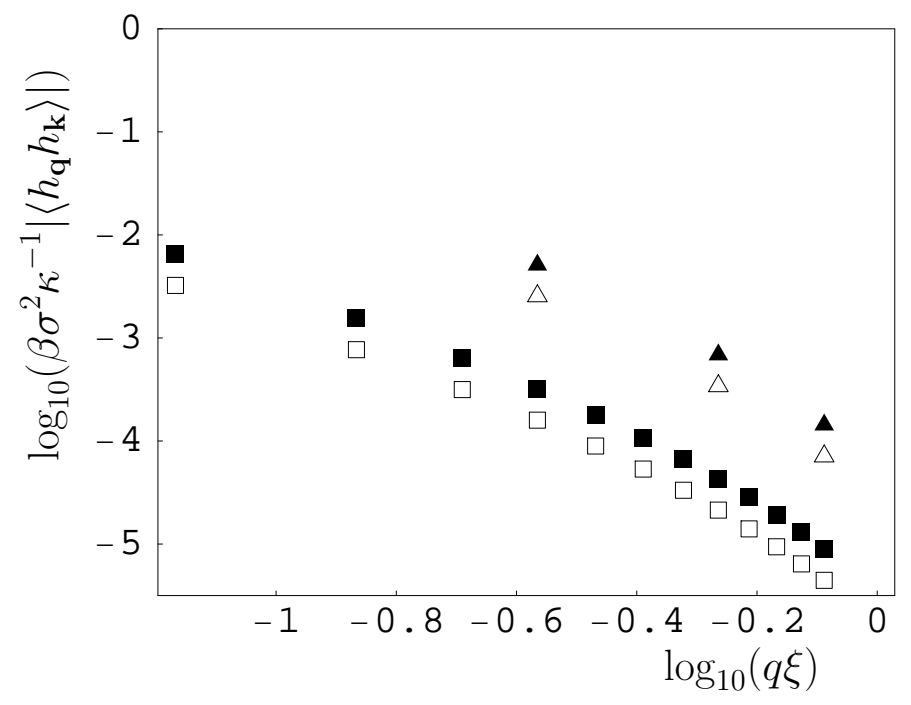

FIG. 4: Non-diagonal fluctuation spectrum $\left\langle h_{\mathbf{q}} h_{\mathbf{k}}\right\rangle$ in the presence of lacking bonds, for $\mathbf{q} \neq-\mathbf{k}$ but $\|\mathbf{q}\|=\|\mathbf{k}\|=q$, as detailed in the text. The squares (resp. triangles) correspond to one missing bond per unit cell, as in Fig. 2 but for $M=80$ (resp. $M=20)$. The values of $q$ are quantified by the periodic boundary conditions. The filled symbols are numerically calculated from Eq. (10), while the open symbols correspond to the analytical approximation discussed in the text.

where $\mathbf{u}$ is the unit vector parallel to the missing bond. In the $q \rightarrow 0$ limit, we obtain

$$
\left|\left\langle h_{\mathbf{q}} h_{\mathbf{k}}\right\rangle\right| \approx \frac{\mu k_{\mathrm{B}} T}{\sigma^{2} q^{2}} \rho,
$$

which shows that the non-diagonal spectrum is proportional to the defect density $\rho=1 /\left(3 M^{2}\right)$.

In conclusion, in the light of this model experimental measurements of RBC equilibrium fluctuations under strain could give new informations concerning the structure and elasticity of the cytoskeleton. Since the defects and the plasticity of RBC's are believed to be controled by the ATPase activity [17], it should also be interesting to investigate the (equilibrium) non-diagonal spectrum at various stages of the erythrocyte life or in particular conditions, such as capillary transport. Finally, it should be possible to study in a Langevin picture the dynamical properties of this model system, thereby determining the consequence of non-equilibrium ATP-driven noise on the fluctuation spectrum. This would be extremely interesting, as recent experiments reveal strong non-equilibrium effects [17, 22, 23].

\section{Acknowledgments}

We thank Dr. David Lacoste for stimulating discussions at the early stage of this work and Prof. Ken Sekimoto for precious comments and fuitfull discussions all along.

[1] B. Alberts, A. Johnson, J. Lewis, M. Raff, K. Roberts, P. Walter, Molecular Biology of the Cell (Garland, New York, 2002), 4th ed.

[2] Statistical Mechanics of Membranes and Interfaces, D. R. Nelson, T. Piran, S. Weinberg eds. (World Scientific, Singapore, 1989).

[3] S. A. Safran, Statistical Thermodynamics of Surfaces, Interfaces, and Membranes (Addison-Wesley Publishing Company, 1994).

[4] O. G. Mouritsen, Life-as a matter of fat (The frontiers collection, Springer, Berlin, 2005).

[5] T. J. Byers and D. Branton, Proc. Natl. Acad. Sci. USA 82, 6153 (1985).

[6] V. Bennett, Biochim. Biophys. Acta 988, 107 (1989).

[7] N. Mohandas and E. Evans, Annu. Rev. Biophys. Biomol. Struct. 23, 787 (1994).

[8] S. K. Boey, D. H. Boal, and D. E. Discher, Biophys. J. 75, 1573 (1998), Biophys. J. 75, 1584 (1998).

[9] D. H. Boal, Biol. Bull. 194, 331 (1998).

[10] G. H. W. Lim, M. Wortis and R. Mukhopadhyay, PNAS 99, 16766 (2002). 
[11] R. Mukhopadhyay, G. H. W. Lim and M. Wortis, Biophys. J. 82, 1756 (2002).

[12] G. Lenormand, S. Henon, A. Richert, J. Simeon and F. Gallet, Biophys. J. 81, 43 (2001).

[13] A. Zilker, H. Engelhardt and E. Sackmann, J. Physique 48, 2139 (1987).

[14] N. Gov, A. G. Zilman, and S. A. Safran, Phys. Rev. Lett. 90, 228101 (2003).

[15] J.-B. Fournier, D. Lacoste and E. Raphaël, Phys. Rev. Lett. 92, 018102 (2004).

[16] N. Gov and S. A. Safran, Phys. Rev. E 69, 011101 (2004).

[17] N. Gov and S. A. Safran, Biophys. J. 88, 1859 (2005).

[18] H. S. Seung and D. R. Nelson, Phys. Rev. A 38, 1005 (1988).

[19] W. Helfrich, Z. Naturforsch. C 28, 693 (1973).

[20] P.-G. de Gennes, Scaling concepts in polymer physics (Cornell University Press, London, 1986).

[21] Y. Kantor and D. R. Nelson, Phys. Rev. Lett. 58, 2774 (1987).

[22] S. Tuvia et al., PNAS 94, 5045 (1997).

[23] S. Tuvia, S. Levin, A. Bitler, and R. Korenstein, J. Cell. Biol. 141, 1551 (1998).

[24] B. W. Shen, R. Josephs, and T. L. Steck, J. Cell. Biol. 102, 997 (1986).

[25] H. Strey, M. Peterson, and E. Sackmann, Biophys. J 69, 478 (1995).

[26] For an array of defects, however, there will be (weak) extinction conditions due to the translational symmetry.

[27] C. Dubus and J.-B. Fournier, in preparation. 